\title{
COVID-19 in Healthcare Workers of Dedicated COVID-19 Screening OPD: A Case Series Based Study from AIIMS, New Delhi, India
}

\section{Pankhuri Dudani ${ }^{1}$, Bharathi Arunan ${ }^{2}$, Vikas, H. $^{3}$, Arvind Kumar ${ }^{4}$, Piyush Ranjan ${ }^{5}$, Upendra Baitha ${ }^{6}$, Prayas Sethi ${ }^{6}$ and Naveet Wig $^{7}$}

${ }^{1}$ Resident, Department of Dermatology, ${ }^{2}$ Resident, Department of Medicine, ${ }^{3}$ Assistant Professor, Department of Hospital Administration, ${ }^{4}$ Associate Professor, Department of Medicine, ${ }^{5}$ Additional Professor, Department of Medicine, ${ }^{6}$ Assistant Professor, Department of Medicine, ${ }^{7}$ Professor and Head, Department of Medicine. All India Institute of Medical Sciences, New Delhi, India

Keywords: COVID screening OPD, Health care workers, Standard Precautions

\section{Corresponding author}

Arvind Kumar

Associate professor, Department of Medicine, 3094A, third floor, Teaching

Block, AIIMS, New Delhi-110029

Email: linktoarvind@gmail.com

\begin{abstract}
$\mathrm{T}$ o keep up with the current COVID-19 pandemic, the healthcare infrastructure is continually changing and growing at a rapid pace. Protection of our frontline workers is of utmost priority. Here, we describe the measures taken at our tertiary care center for this purpose. A dedicated COVID-19 screening centre was setup for healthcare workers (HCW), run by frontline HCWs themselves. Over the 6 months that this OPD has run, it has catered to hundreds of HCWs, with a small number of staff themselves contracting COVID-19. The individual cases have been enumerated, and the changes implemented from lessons we learnt have been discussed.
\end{abstract}

Management of any pandemic requires adequately-staffed hospitals and an evolving infrastructure. The front-line workers are at the forefront of response in assessing and managing patients. This puts them at a high risk of infection. There are clear publically available guidelines to reduce risk to HCWs at workplace; but implementability varies depending on the setting.

Worldwide, HCWs remain a significantly affected group. In Netherlands, within 10 days of the first reported case, $5 \%$ of $1497 \mathrm{HCWs}$ had tested positive in different hospitals (Sikkema et al. 2020). Recently, over 15\% of COVID-19 patients in Victoria, Australia were reported to be HCWs. Early data from Wuhan, China indicated that incidence in HCW was $144.7 / 10^{6}$, compared with $41.7 / 10^{6}$ among general population, with $29 \%$ of hospitalized patients being the former. Italian data reflected that at one point, $20 \%$ of all HCWs were COVID-19 positive (Saglietto et al. 2020). With newer testing methods including antibody-testing available, retrospective diagnosis could be made as well. A large hospital in Spain demonstrated a cumulative (past and present) infection rate of $11.2 \%$ among its healthcare workers, $40 \%$ of whom had been asymptomatic. The odds of being seropositive were higher with larger households, indicating that household contact may be an important source of infection for a HCW; and no increased risk with working in COVID unit was found, probably due to increased caution and adherence to safety regulations. A survey of $18,000 \mathrm{HCW}$ in India found flu-like symptoms in 14.7\% HCWs, while 1.8\% tested positive for the virus. The positivity rate was not found to be associated with high-risk area of work, and no difference was observed between those given HCQS prophylaxis and those who were not (Jha et al. 2020). A case control study from India, however, found 
a dose-response relationship between hydroxychloroquine (HCQ) prophylaxis and contraction of COVID infection among HCWs (Chatterjee et al. 2020).

A higher risk of infection has been observed- A prospective observational study of front-line healthcare workers in the US found them to be at a three-five times increased risk of COVID-19 infection even after correcting for possible biases of repeated testing. This was seen more with reused/ inadequate personal protective equipment (PPE), but was also seen in those with adequate PPE and those reporting no contact with known COVID-19 positive patients. A population-based cohort study in US and UK including 2,035,395 community individuals and 99,795 HCWs found the hazard ratio of a positive test to be 11.6 for frontline HCWs when compared with general population (Nguyen $e t$ al. 2020). A rapid review found insufficient/ inadequate PPE to be the most important risk factor, followed by working in high-risk areas, longer working hours, limited knowledge of infection control as well as lack of established hospital infection control practices or poor adherence to them. An early study also found association with suboptimal hand hygiene (Ran et al. 2020).

In our tertiary care hospital in North India, we have a dedicated COVID-19 screening Outpatient department (OPD) for healthcare workers and their families, supported by a virology and serology laboratory as well as a contacttracing team. This OPD was planned and opened early on in the pandemic to cater particularly to the frontline HCWs of our institution. The OPD was designed to ensure a lowrisk environment for the health care workers which included a plexiglas shield measuring 7 feet from the ground to sequester the screening staff and kiosks with rubber gloves to ensure safety of the workers involved in sampling patients. Level 3 PPE kits were available for sampling of children/ other patients who could not be sampled at the kiosks. Fresh N95 masks were given to all staff daily. Patient contact was thus minimized. Additionally, the staff was rotated every 4 weeks and provided appropriate quarantine period before resuming duties elsewhere.

In the period of two months between April and June 2020, 6 workers of the employee health scheme (EHS) COVID screening OPD (2 doctors and 4 auxiliary workers) contracted COVID. The details as follows:

\section{Case 1}

A 25 year-old resident doctor developed mild headache and coryza, 2 weeks after starting work at this OPD, and was found to be COVID-19 positive. As she lived alone and walked to work, the source of infection was suspected to be from the OPD itself. Her work consisted of handing out testing kits to the patients. Due to a high Plexiglas barrier, she had to step out of the enclosure multiple times to hand these kits to patients. As these were early days in the foundation of the OPD, the HCWs were provided only N-95 masks and gowns. She was home isolated,and advised routine monitoring of temperature and oxygen saturation. She had mild symptoms with 8 days of fever. After the first 3 weeks, she developed severe myalgia and arthralgia, along with mild respiratory discomfort at rest. Examination then revealed normal respiratory rate and saturation, but tachycardia with minimal activity and low diastolic blood pressure (with preserved MAP). Routine tests found an elevated ESR and mild transaminitis. At 3 months of follow up, she had improvement from baseline symptoms but reported a reduced work capacity and persistent myalgia. She had 3 high-risk contacts, but they were asymptomatic, and none of them tested positive for COVID at day 8.

\section{Case 2}

A 26 year-old female data entry operator tested positive 1 week after starting work at the EHS screening OPD. She had developed high-grade fever, and 2 more members of her 5-membered family developed similar symptoms simultaneously. They tested positive for COVID-19, following which they were admitted at our hospital's COVID facility. As she worked behind the shield at all times, lived with her family and travelled in a bus with other employees of the hospital, she was suspected to have acquired the infection from outside the workplace. She had high-grade fever for next 5 days, along with anosmia and aguesia. She has subsequently recovered with minimal sequalae.

\section{Case 3}

A 27-year old male resident doctor developed fever, myalgia, sore throat and mild cough, 8 days after finishing 8 weeks of working in EHS OPD. He was involved in talking to patients and sampling patients at the kiosk. He lived alone. The source of infection was suspected to be the workplace itself. He was admitted for 10 days, the stay being uneventful. He had one high-risk contact who tested negative.

\section{Case 4}

A 27 year-old male technician working in sample collection 
at the kiosks developed fever 4 weeks after working in this OPD. He lived with his mother, and used to travel to work alone on his two-wheeler, and had no other contacts. The source was suspected to be the workplace. He was admitted in our COVID facility for 10 days, with an uneventful recovery.

\section{Case 5}

A 25 year-old paramedical worker developed fever 2 weeks after starting work at our OPD. Similar to our first patient, her work included handing out testing kits to suspected patients, for which she would step out of the Plexiglas enclosure multiple times during her shift. She stayed with her family and was travelling to and from work via the bus shuttle. The source of infection could not be determined. She was also admitted in our COVID facility.

\section{Case 6}

A 30-year-old hospital attendant tested COVID positive after 6 weeks of working at the OPD. He would man the gate and implement social distancing among the patients waiting their turn, and would sometimes come within 1 meter distance. He was provided with an N 95 mask along with a face shield and gown during his shifts. The source of infection could not be ascertained.

\section{DISCUSSION}

Following these infections, and as we learnt more with the evolving pandemic, many changes were made in the functioning of the OPD. On retrospection, it was realized that the place of acquiring the infection is difficult to determine, whether it was workplace or outside. However, breach in the standard precautions is a must for acquiring COVID-19 infection, independent of the place of acquiring the infection. Few of the factors which were observed at this COVID facility were: handing out the testing kits to the OPD attenders in person without appropriate PPE; the sudden surge in the number of cases attending our center, due to which maintaining adequate social distancing was not strictly followed; the laxity in behavior towards strict use of N95 mask or infrequent breach, inappropriate COVID related behavior of the users at waiting time, inappropriate exchange of OPD cards/notes between the user and OPD staff through the glass shield inside the enclosure area and education and training of the staff posted in the OPD was also incomplete in early days of pandemic as it definitely needed repetition and regular re-enforcement.

To address these issues, a slot was made in the Plexiglas through which kits were passed on to the OPD patients. This ensured minimal contact between OPD patients and HCWs. Mike systems were also installed in the Plexiglas for effortless communication. A token system was started to deal with the increasing crowds, as the footfall in the OPD had increased from 50 a day to more than 300 a day in a span of a week. This system ensured that the crowd in the OPD could be controlled adequately and social distancing could be maintained between the patients themselves, as well as between patients and HCWs, specially patient-care coordinators and security staff. Additionally, all staff posted in the OPD were regularly educated regarding appropriate use and disposal of PPE, screening for symptoms, social distancing practices and frequent hand-washing, which at every stage in the pandemic have been proven to be one of the best ways of preventing COVID-19 infection. The task of education and training of the health care workers was taken up by Hospital infection control committee in coordination with the faculty in-charges from department of Medicine and Hospital administration of the COVID OPD facility under the supervision of the institutional COVID task force chaired by Head of the department of Medicine. For monitoring the quality of precautions the HCWs at the COVID-19 OPD duty had made their own self-help group in each work shift to observe each other's COVID inappropriate behavior and rectify them in a conducive environment.

The observations after implementation of the above mentioned changes have been satisfactory; only one staff of EHS OPD tested positive in the next three months. We believe that improving the workplace safety is a continuous process requiring one to constantly look for possible lacunae. Our experience has been encouraging, as appropriate changes were promptly made and found to improve workplace safety. The number of infections reduced significantly, despite the increased community transmission with reversal of lockdown and increased number of new cases. The HCWs who did contract the infection had a mild course of disease. Support of administration was imperative to creation of a safe workplace, and this has been found to be instrumental in other parts of the world too (Delgado-Gallegos et al. 2020). The CDC has also called for ensuring HCW education, encouraging daily self-screening of symptoms, and nonpunitive and flexible policies of sick-leave for self-isolation and quarantine. 


\section{No Disclosure}

\section{No Conflict of interest}

\section{REFERENCES}

Chatterjee, P., Anand, T., Singh, K.J., Rasaily, R., Singh, R., Das, S., Singh, H., Praharaj, I., Gangakhedkar, R.R., Bhargava, B. and Panda, S. 2020. Healthcare workers \& SARS-CoV-2 infection in India: A case-control investigation in the time of COVID-19. Indian J. Med. Res., 151: 459-467.

Jha, S., Soni, A., Siddiqui, S., Batra, N., Goel, N., Dey, S., Budhiraja, S. and Naithani, R. 2020. Prevalence of Flulike Symptoms and COVID-19 in Healthcare Workers from India. J. Assoc. Physicians India, 68: 27-29.

Nguyen, L.H., Drew, D.A., Joshi, A.D., Guo, C.G., Ma, W., Mehta, R.S.etal.2020.Risk of COVID-19 among frontlinehealthcare workers and the general community: a prospective cohort study. Med. Rxiv., [Preprint]. 2020:2020.04.29.20084111. doi: 10.1101/2020.04.29.20084111. Update in: Lancet Public Health. 2020 Jul 30;: PMID: 32511531; PMCID: PMC7273299.
Ran, L., Chen, X., Wang, Y., Wu, W., Zhang, L., Tan, X. 2020. Risk Factors of Healthcare Workers with Coronavirus Disease 2019: A Retrospective Cohort Study in a Designated Hospital of Wuhan in China. Clin. Infect. Dis., 71: 2218-2221.

Saglietto, A., D’Ascenzo, F., Zoccai, G.B. and Ferrari, G.M.D. 2020. COVID-19 in Europe: the Italian lesson. The Lancet, 395: $1110-1$.

Sikkema, R.S., Pas, S.D., Nieuwenhuijse, D.F., O’Toole, Á., Verweij, J., Linden A van der, et al. 2020. COVID-19 in health-care workers in three hospitals in the south of the Netherlands: a cross-sectional study. Lancet Infect Dis., 20: 1273-80.

Delgado-Gallegos, J.L., Montemayor-Garza, R. de, J., PadillaRivas, G.R., Franco-Villareal, H. and Islas, J.F. 2020. Prevalence of Stress in Healthcare Professionals during the COVID-19 Pandemic in Northeast Mexico: A Remote, Fast Survey Evaluation, Using an Adapted COVID-19 Stress Scales. Int. J. Environ. Res. Public Health, 17: 7624. 